\title{
Royal road from geometric to discrete optics
}

\author{
Kurt Bernardo Wolf* \\ Instituto de Ciencias Físicas, Universidad Nacional Autónoma de México, Av. Universidad s/n, \\ Cuernavaca, Mor. 62210, México
}

Received January 08, 2015; accepted March 16, 2015; published March 31, 2015

\begin{abstract}
There are well-trodden paths between geometric and wave optics while, with sampling and interpolation, a further bridge to discrete optics is usually traversed. Our previous work points to a Royal road to link the paraxial, metaxial, and global geometric with discrete models, where canonicity becomes unitarity and where phase spaces retain thei meaning. This short review maps the terrain that has been traversed.
\end{abstract}

The line of work of the Óptica Matemática projects centres on the applications of group theoretical methods to geometric, wave and discrete (mostly finite) optical models, although it still lies on the fringes of the mainstream applied research in Mexico. We use the fact that when two models exhibit the same underlying symmetry group, a correspondence can be established between them. In this contribution to the monographic Special Issue we give an overview of the common symmetries and distinct realizations in the regimes of geometric and discrete optics.

The geometric model of optics assumes "screens" on which a manifold of rays is characterized by their position $\mathbf{q}$ and their momentum $\mathbf{p}$ (i.e. $|\mathbf{p}|=n \sin \theta$, with the index of refraction $n$ and angle $\theta$ to the screen normal, allowing for $\mathbf{p} \in \mathrm{R}^{2}$ in the paraxial régime), which satisfy the basic Poisson brackets $\left\{q_{i}, p_{j}\right\}=\delta_{i, j}$. Optical transformations $\mathbf{q}$ $\rightarrow \mathbf{q}^{\prime}(\mathbf{q}, \mathbf{p}), \mathbf{p} \rightarrow \mathbf{p}^{\prime}(\mathbf{q}, \mathbf{p})$ due to free flight, refracting surfaces, or transit through inhomogeneous media, can only be canonical, i.e., preserve the Poisson brackets [1, ch. 3]. Canonical transformations are reversible and, since no rays are gained nor lost, they conserve information; caustics are not singularities in a phase space. In the linear (paraxial) regime, these transformations form the real symplectic group $S p(2 D, \mathrm{R})$ in $D$ dimensions. Light fields are understood as generally complex functions $\varrho(\mathbf{q}, \mathbf{p})$, whose absolute square can represent intensity.

The discrete model of optics that we use consists of finite pixelated screens, where each pixel contains a complex value of the discrete wavefunction that forms the image. The pixel address is given by the equally-spaced finite spectrum of a position operator $\mathbf{Q}$, and a momentum operator is defined through $\mathbf{P}:=\mathrm{i}[H, \mathbf{Q}]$, with the Lie bracket (commutator) of the generator $H$ of the FourierKravchuk transform [2] (i.e. of a harmonic oscillator other models with infinite discrete screens use the

*E-mail: bwolf@fis.unam.mx repulsive oscillator or the free system [3-4]). The resulting structure is the unitary Lie algebra $s u(2)$ for $1 \mathrm{D}$ screens, and $s u_{x}(2) \times s u_{y}(2)=s o(4)$ for $2 \mathrm{D} N_{x} \times N_{y}$ screens. This Lie algebra exponentiates to the corresponding unitary Lie groups of transformations. An important property of the discrete model is that, when the number and density of pixels increases without bound, it contracts to the common Heisenberg-Weyl algebra and its quantum-mechanical or paraxial wave-optical rendering of operators and wavefields, which in turn come down to the classical or geometric model when the wavelength tends to zero.

In $1 \mathrm{D}$, this formalism leads to the non-standard commutation relation $[Q, P]=\mathrm{i} J_{3}$, with $J_{3}:=H-j-1 / 2$, that characterizes the representation of dimension $N=$ $2 j+1$ of $s u(2)$, and provides the Hamilton equations of the harmonic oscillator in the form $[H, Q]=-\mathrm{i} P$ and $[H, P]=$ i $Q$, thus identifying $Q=: J_{1}$ and $P=: J_{2}$, with the position and momentum operators. The position spectrum is $\left.m\right|_{-j} ^{j}$ in the $Q$ eigenbasis, and $f_{m}=(m \mid f)$ are the values of any pixelated $1 \mathrm{D}$ image; a $\pi / 2$ rotation generated by $H$ yields, through the Fourier-Kravchuk transform, the discrete and finite momentum eigenbasis of $P$ [2]. To this algebra we can add a fourth generator, 1 , that will generate overall phases, and turn the algebra into $u(2)=u(1) \times s u(2)$. This $u(1)$ does not have a counterpart in classical models because $\{1, \circ\}=0$.

In $2 \mathrm{D}$, there are two position and momentum operators, whose postulated algebraic relations are $\left[Q_{x}, Q_{y}\right]=$ : i $M:=$ $\left[P_{x}, P_{y}\right]$, defining an new (angular momentum) operator $M$, which closes together with them and $H$ through $[M$, $\left.Q_{x}\right]=+\mathrm{i} Q_{y},\left[M, Q_{y}\right]=-\mathrm{i} Q_{x},\left[M, P_{x}\right]=+\mathrm{i} P_{y},\left[M, P_{y}\right]=-\mathrm{i} P_{x}$, and $[M, H]=0$, into the Lie algebra $s u_{x}(2) \times s u_{y}(2)=s o(4)$. In the same way, a 2D image is a matrix of values $f_{m x, m y}=$ $\left(m_{x}, m_{y} \mid f\right)$ in Cartesian coordinates on a rectangular screen.

For $N_{x}=N_{y}$, a similar structure allows also for screens with circular pixellation [5], where radial and angular coordinates are determined using the sub-algebra chain $s o(4)>s o(3)>s o(2)$. The map between Cartesian and circular pixellations is unitary, so it is reversible and no information is lost in the transfer of images between the two. Including overall phases, the $2 \mathrm{D}$ algebra is $u_{x}(2) \times u_{y}(2)=u(1) \times u(1) \times s o(4)$. 
The Fourier group is a maximal compact subgroup of the real symplectic group of linear canonical transformations. In $1 \mathrm{D}$, this is the $u(1)<s p(2, \mathrm{R})$ that generates phase space rotations in the geometric model, and the fractional Fourier-Kravchuk transform in the discrete one. In $2 \mathrm{D}$, it is the four-parameter $u(2)<s p(4, \mathrm{R})$ that generates rotations within the $4 \mathrm{D}$ phase space, composed of rotations in the $x$ - and $y$ - phase subplanes which are fractional Fourier-Kravchuk transforms in discrete optics; cross-rotations in the $q_{x}-p_{y}$ and $p_{x}-q_{y}$ planes which are gyrations in wave optics and in discrete optics as well [6]; and rotations in the $x-y$ plane of image and momentum, which are natural in geometric optics, and are thus imported onto square pixelated screens [5], as unitary and fully reversible transformations [7].

Free flights, thin lenses and squeezings are beyond the Fourier group that is strictly the same for both the paraxial geometric and discrete optical models. We recall that in $2 \mathrm{D}$, the symplectic group $S p(4, \mathrm{R})$ has 10 parameters; the remaining six in geometric optics include slantings of phase space, generated by the Poisson operators $\left\{p_{x}{ }^{2}, \circ\right\},\left\{p_{y}{ }^{2}, \circ\right\}$ (free flights), $\left\{q_{x}^{2}, \circ\right\},\left\{q_{y}^{2}, \circ\right\}$ (thin lenses), and $\left\{p_{x} q_{x}, \circ\right\},\left\{p_{y} q_{y}, \circ\right\}$ (squeezings). What are their counterparts in discrete optics? There we have to resort to the covering of the 6-parameter algebra $s u_{x}(2) \times s u_{y}(2)$, namely the squares of the position and momentum generators identified within each factor. Since in discrete optics on square screens these are represented by $N^{2} \times N^{2}$ self-adjoint matrices, their squares and higher powers are still self-adjoint matrices that exponentiate to unitary matrices which will act on the pixelated image field. The Hamiltonian generators $H_{x}$ and $H_{y}$ need not be considered beyond their first power because the Casimir operators relate their sum of squares to the squares of the previous ones.

The metaxial regime of geometric optics in 1D is generated by Poisson operators $\left\{p^{r} q^{s}, \circ\right\}$, for $r+s>2$. These correspond in the discrete model with matrix representations of the covering algebra of $s u(2)$, which are selfadjoint and of dimension $N \times N$. Since there are no more than $N^{2}$ independent self-adjoint complex matrices that exponentiate to the group $U\left(N^{2}\right)$, no more than $N^{2}-4$ aberrations exist in the discrete model (removing the 3 linear transformations and the overall phase). The correspondence between the two models has been defined through the matrices $\left\{\mathbf{P}^{r} \mathbf{Q}^{s}\right\}_{\text {Weyl }}$, using the Weyl ordering of the factors (which preserves self-adjointness) -but now we also have a second set of aberration generators $\left\{\mathbf{P}^{r} \mathbf{Q}^{s} \mathbf{H}\right\}_{\text {Weyl }}$ that include a harmonic oscillator-like factor [8]. We can check by counting that these exhaust the Lie algebra $u\left(N^{2}\right)$. The 2D case has not yet been pursued beyond unitary rotations [5] and gyrations [6], but its computational treatment is presently within the Óptica Matematica research project.
We should remark that, even in 1D, the full $U\left(N^{2}\right)$ group includes not only "continuous" transformations such as those afforded by optical setups with all aberrations properly controlled, but also all pixel permutations. The permutation of two pixels, $i$ and $j$, lies within a $S O(2)$ subgroup that rotates between the $i^{\text {th }}$ and $j^{\text {th }}$ rows of the image vector. It is thus not clear whether the discrete models are in fact much "larger" than their physically possible geometric counterparts.

A last and sobering remark on the algorithms for unitary transformations of pixelated screens is in order: they are computationally the slowest algorithms of all, because generally every transformed pixel value depends on the values of all the original pixels, since the $N \times N$ matrices in the group representation are irreducible for 1D, and reducible $N^{2} \times N^{2}$ (but not in the pixel basis) for $2 \mathrm{D}$. This is true in particular with rotations, where the commercial algorithms that rotate digital images use any one of several interpolation strategies, as can be seen in MATHEMATICA ${ }^{\circ}$.

The $s u(2)$ Wigner function defined in [9] maps 1D finite signals onto a compact phase space that is a sphere in an ambient 3D space, and has the desired properties of covariance with the $s u(2)$ model of discrete optics. It serves to visualize the action of aberrations on signals [8], and to identify translated coherent states with their rotated discrete counterparts [10]. Here also, when $s u(2)$ contracts to the Heisenberg-Weyl algebra, the radius of the sphere grows and the Wigner function becomes the standard one on the phase plane. With these tools, in Ref. [8] we pictured the linear transformations and aberrations acting on a "rectangle" signal of 0's and 1's. For 2D it does not seem convenient to interpret a pixelated image on a 4sphere within an ambient 6D space; although the direct product nature of $s u_{x}(2) \times s u_{y}(2)$ can be exploited, we have not yet studied this possibility.

The $4 \pi$ regime of optics requires a different group of symmetries to describe the geometric and build a discrete model of optics. No longer bound to 2D screens, we consider the iso(3) Euclidean symmetry algebra that generates translations and rotations in space. In [1, App. B] we showed that this algebra contains several optical models for lines, planes, bands, screws, etc., and also a sui generis Hilbert space for the monochromatic Helmholtz wave-fields. The 2D model of the latter has a continuous compact basis (a circle) of plane waves, and also an infinite discrete basis of a line of $J_{0}(z)$ and $J_{1}(z) / z$ Bessel functions, placed a half-wavelength apart [4], [11]. For the latter, the 1D case has been examined through its iso(2) Wigner function depicted on a cylinder [13].

Another more recent approach to the $4 \pi$ régime is based on the geometric optical Maxwell fish-eye, which is well known to be the stereographic projection of free motion on a sphere [1, ch. 6], and where geometric rays are circles. The wave-optical version uses the spherical 
harmonic basis to define new bases of position and momentum characterized for having maximal second moment, and whose contraction, when the radius of the sphere grows without bound, is the Helmholtz medium, matching with the bases mentioned in the previous paragraph [14]. Their examination through the so(3) and iso(2) Wigner functions is also one of our current aims.

In conclusion, we can state that our interest lies building and studying discrete models of optics that can be set up directly from the geometric picture, and whose contraction (when the number and density of pixels grow) yields a useful wave-optical model, which in turn will contract to a geometric one). One reason for this strategy is that approximations by a finite set of values will converge smoothly to the intended continuous wavefields. Another reason is that other models (such as lines, planes, screws, etc.) can be handled with the same tools. But the main reason may simply be the quest to find symmetries behind the objects and systems in Nature. Along that Royal road we also find interesting relations between discrete special functions (Kravchuk, Meixner, Pollaczek, Hahn) and their relation with a well-known group of theoretical constructs, such as Wigner's and Bargmann's little-d functions as well as the Clebsch-Gordan coefficients, dressed in the guise and garb of optics.

Since the fields recounted here include the collaboration of many colleagues and span many years, let me thank my numerous co-authors, many of whose independent works I have not quoted here for editorial reasons. I also thank my present students (M.C. Salto-Alegre, A.R. Urzúa, and R.K. Uriostegui) and the indispensable help of $G$. Krötzsch for illustration. The Óptica Matemática projects were supported by the funds PAPIIT-UNAM IN101115 and SEP-CONACyT 79899, to which I extend my recognition.

\section{References}

[1] K.B. Wolf, Geometric Optics on Phase Space (Heidelberg, SpringerVerlag 2004).

[2] N.M. Atakishiyev, K.B. Wolf, J. Opt. Soc. Am. A 14, 1467 (1997).

[3] C.A. Muñoz, J. Rueda-Paz, K.B. Wolf, J. Phys. A 42, 485210 (2009).

[4] K.B. Wolf, J. Phys. A 46, 335202 (2013).

[5] N.M. Atakishiyev, G.S. Pogosyan, L.E. Vicent, K.B. Wolf, J. Phys. A 34, 9399 (2001).

[6] K.B. Wolf, T. Alieva, J. Opt. Soc. Am. A 25, 365 (2008).

[7] K.B. Wolf, L.E. Vicent, SIGMA 7, 053 (2011), http://www.emis.de/journals/SIGMA/S4.html

[8] K.B. Wolf, J. Phys. A 41, 304026 (2008).

[9] N.M. Atakishiyev, S.M. Chumakov, K.B. Wolf, J. Math. Phys. 39, 6247 (1998).

[10] K.B. Wolf, Science 4, 141 (2010).

[11] P. González-Casanova, K.B. Wolf, Numer. Meth. Part. Diff. Eqs. 11, 77 (1995).

[12] L.M. Nieto, N.M. Atakishiyev, S.M. Chumakov, K.B. Wolf, J. Phys. A 31, 3875 (1998).
[13] C. Salto-Alegre, K.B. Wolf, Position and momentum bases on the sphere for the monochromatic Maxwell fish-eye. Accepted in: Open Access J. Phys. Conf. Series (JPCS), Ed. by Joris Van der Jeugt. Proceedings of the $30^{\text {th }}$ International Colloquium on Group Theoretical Methods in Physics (Ghent, Belgium, 14-18 July, 2014). 